\title{
APPLICATION OF UNCERTAIN VARIABLES TO DECISION MAKING IN A CLASS OF DISTRIBUTED COMPUTER SYSTEMS
}

\author{
Z. Bubnicki \\ Institute of Control and Systems Engineering \\ Wroclaw University of Technology \\ Wyb. Wyspianskiego 27, 50-370 Wroclaw, POLAND \\ email: bubnicki@ists.pwr.wroc.pl
}

Abstract: The paper is concerned with a class of uncertain distributed systems described by a set of relations containing unknown parameters. The purpose of the paper is to present an application of so called uncertain variables to decision making in the systems under consideration. In the first part of the paper, a problem of the decision making in the distributed system in which the knowledge of the system as a whole is decomposed into two levels is considered. In the second part it is shown how to apply the uncertain variables in the determination of computational tasks allocation in a set of parallel processors.

Key words: distributed systems, parallel processing, uncertain systems

\section{INTRODUCTION}

The paper concerns a class of distributed systems described by a set of relations between the variables characterizing the decisions and their effects (a relational knowledge representation). For the decision making in such a class of systems the algorithms of learning have been proposed [1]. The purpose of this paper is to present an application of so called uncertain variables to the decision making in the same class of distributed systems. The uncertain variable $\bar{x}$ is defined by a certainty distribution $h_{x}(x)=v(\bar{x} \widetilde{\epsilon} x)$, i.e. the certainty index $v$ that " $\bar{x}$ is approximately equal to $x$ ". The certainty distribution is given by an expert and describing his/her knowledge of the approximate values of the variables $[2,3,4]$. 


\section{DECISION MAKING PROBLEM}

Let us consider a distributed system with a structure presented in Fig.1 where $u_{j} \in U_{j}, x_{j} \in X_{j}(j \in \overline{1, k}), y \in Y$ are real number vectors. The lower and upper level subsystems are described by relations

$$
\begin{aligned}
& R_{j}\left(u_{j}, x_{j} ; c_{j}\right) \subseteq U_{j} \times X_{j}, \quad j \in \overline{1, k} \\
& R\left(x_{1}, \ldots, x_{k}, y ; b\right) \subseteq X_{1} \times \ldots \times X_{k} \times Y,
\end{aligned}
$$

respectively. The set of relations forms the knowledge representation of our system with the input $u=\left(u_{1}, \ldots, u_{k}\right)$ (relational knowledge representation). The unknown parameters $b$ and $c_{j}$ are assumed to be values of uncertain variables $\bar{b}$ and $\bar{c}_{j}$ described by the certainty distributions $h_{b}(b)$ and $h_{c j}\left(c_{j}\right)$, respectively. The decision problem consists in finding $u^{*}$ maximizing the certainty index that the set of all possible $y$ approximately belongs to the set $D_{y} \subset Y$ given by a user. The problem may be decomposed into two levels.

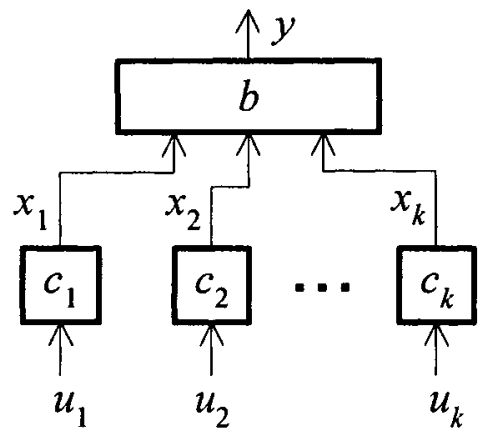

Figure 1. Two-level system

1. Upper level problem: Given $R\left(x_{1}, \ldots, x_{k}, y ; b\right), h_{b}(b)$ and $D_{y}$, find

$$
x^{*}=\arg \max _{x} v\left[D_{y}(x ; \bar{b}) \widetilde{\subseteq} D_{y}\right] \triangleq v(x)
$$

where $x=\left(x_{1}, \ldots, x_{k}\right)$ and $D_{y}(x ; \bar{b})$ is the set of all possible $y$ for the fixed 
$x$. It may be shown $[1,2]$ that

$$
v(x)=\max _{b \in D_{b}(x)} h_{b}(b)
$$

where $D_{b}(x)=\left\{b \in B:(x, y) \in R(x, y ; b) \rightarrow y \in D_{y}\right\}$.

2. Lower level problem: Given $R_{j}\left(u_{j}, x_{j} ; c_{j}\right), h_{c j}\left(c_{j}\right)$ and $D_{x j}$, find $u_{j}^{*}$ maximizing $v\left(\bar{x}_{j} \tilde{\epsilon} D_{x j}\right) ; j \in \overline{1, k}$. The sets $D_{x j}$ are such that $D_{x 1} \times D_{x 2} \times \ldots \times D_{x k} \subseteq D_{x}$ where $D_{x}$ is the set of solutions $x^{*}$ of the upper level problem. The solution is analogous to that for the upper level.

\section{TASK ALLOCATION}

The approach presented in the previous section may be applied to a problem of the tasks allocation in the group of parallel processors with uncertain execution times. We assume that the global computational task to be distributed may be decomposed into $N$ separate parts (programs or parts of programs) which may be executed simultaneously by the separate processors. Each partial task is characterized by an upper bound of the execution time $\tau_{j}$ for $j$-th processor $(j=1,2, \ldots, k)$, and $\tau_{j}$ is assumed to be the same for each partial task. The decision problem consists in the determination of the numbers of the partial tasks $n_{1}, n_{2}, \ldots, n_{k}$ assigned to the processors taking into account the execution time $T=\max \left\{T_{1}, T_{2}, \ldots, T_{k}\right\}$ where $T_{j}$ is the execution time for $j$-th processor; $n_{1}+\ldots+n_{k}=N$. If $N$ is sufficiently large, we can determine the decisions $u_{j} \in R^{+}$(any positive numbers) and then obtain $n_{j}$ by rounding off $u_{j}$ to the nearest integer. To apply the notation in the previous section let us denote $T_{j} \triangleq x_{j}, \quad T \triangleq y, \quad \tau_{j} \triangleq c_{j}$. Then the knowledge representation of our distributed system is as follows: $x_{j} \leq c_{j} u_{j}, y=\max \left\{x_{1}, x_{2}, \ldots, x_{k}\right\}$. The unknown parameters $c_{j}$ are assumed to be values of uncertain variables $\bar{c}_{j}$ described by the certainty distributions $h_{c j}\left(c_{j}\right)$ given by an expert estimating the execution times for the partial tasks. The decision problem may be formulated as follows: Given $\alpha$ and $h_{c j}\left(c_{j}\right), j \in \overline{1, k}$, find the 
allocation $u^{*}=\left(u_{1}^{*}, u_{2}^{*}, \ldots, u_{k}^{*}\right)$ maximizing the certainty index of the property: "the user's requirement is approximately satisfied ", i.e. " the approximate value of the execution time $T=y$ is less than $\alpha$ ". Then

$$
u^{*}=\arg \max _{u} v(T \widetilde{\leq} \alpha)
$$

where "T $\widetilde{\leq} \alpha^{\prime \prime}=" D_{T}(u) \widetilde{\subseteq}[0, \alpha] ", D_{T}(u)$ is the set of possible values $T$ for the fixed $u$ and $v[T \widetilde{\leq} \alpha]=v\left[\left(T_{1} \widetilde{\leq} \alpha\right) \wedge \ldots \wedge\left(T_{k} \widetilde{\leq} \alpha\right)\right]=\min v_{j}\left(u_{j}\right)$ where

$$
v_{j}\left(u_{j}\right)=v\left[T_{j} \widetilde{\leq} \alpha\right]=\max _{c_{j} \in D_{c j}\left(u_{j}\right)} h_{c j}\left(c_{j}\right)
$$

and $D_{c j}\left(u_{j}\right)$ is described by the inequality $c_{j} \leq \alpha u_{j}^{-1}$.

The procedure of the determination of $u^{*}$ is then the following:

1. To determine $v_{j}\left(u_{j}\right)$ according to (3). 2. To determine $u_{1}^{*}, u_{2}^{*}, \ldots, u_{k}^{*}$ by solving the maximization problem

$$
\max _{u_{1}, \ldots, u_{k}} \min \left\{v_{1}\left(u_{1}\right), \ldots, v_{k}\left(u_{k}\right)\right\}
$$

subject to constraints $u_{1}+\ldots+u_{k}=N .3$. To round off the values $u_{j}^{*}$ and to obtain the number $n_{j}^{*}$.

Numerical examples and simulations showed a significant influence of the parameters in the certainty distributions on the final results.

\section{NOTES}

This work was supported by the Polish State Committee for Scientific Research under Grant No. 4 T11C 00122

\section{REFERENCES}

1. Bubnicki Z. Knowledge validation and updating in a class of uncertain distributed knowledge systems. Proc. of 16th World Computer Congress, vol. IIP; 2000 August 2125; Beijing. Beijing: Publ. House of Electr. Industry, 2000

2. Bubnicki, $Z$. Uncertain variables and their applications for a class of uncertain systems. International Journal of Systems Science 2001; 32:651-59

3. Bubnicki, $Z$. Uncertain variables and their application to decision making. IEEE Transactions on SMC, Part A: Systems and Humans 2001; 31:587-96

4. Bubnicki, Zdzislaw, Uncertain Logics, Variables and Systems. London, Berlin: SpringerVerlag, 2002 (in press). 